\title{
Chaos and Thermalization in a Dynamical Model of Two Interacting Particles
}

\author{
F.Borgonovi ${ }^{[a, b, c]}$, I.Guarneri ${ }^{[d, b, c]}$, F.M.Izrailev ${ }^{[d, e, f]}$ and G.Casati ${ }^{[d, c, g]}$ \\ ${ }^{[a]}$ Dipartimento di Matematica, Università Cattolica, via Trieste 17, 25121 Brescia, Italy \\ ${ }^{[b]}$ Istituto Nazionale di Fisica Nucleare, Sezione di Pavia, via Bassi 6, 27100 Pavia, Italy \\ ${ }^{[c]}$ Istituto Nazionale di Fisica della Materia, Unità di Milano, via Celoria 16, 20130 Milano, Italy \\ [d] Universitá di Milano, sede di Como, via Lucini 3, 22100, Como, Italy \\ ${ }^{[e]}$ Budker Institute of Nuclear Physics, 630090, Novosibirsk, Russia \\ ${ }^{[f]}$ Instituto de Fisica, Universidad Autonoma de Puebla, Apdo. Postal J-48, Puebla, 72570 Mexico \\ ${ }^{[g]}$ Istituto Nazionale di Fisica Nucleare, Sezione di Milano, via Celoria, Milano, Italy
}

(August 18, 2019)

\begin{abstract}
A quantum dynamical model of two interacting spins, with chaotic and regular components, is investigated using a finite two-particles symmetrized basis. Chaotic eigenstates give rise to an equilibrium occupation number distribution in close agreement with the Bose-Einstein distribution despite the small number of particles $(n=2)$. However, the corresponding temperature differs from that derived from the standard Canonical Ensemble. On the other side, an acceptable agreement with the latter is restored by artificially randomizing the model. Different definitions of temperature are then discussed and compared .
\end{abstract}

PACS numbers: $05.45 .+\mathrm{b}$

The concept of quantum ergodicity, and its connections with the foundations of statistical mechanics, have a long history which, in the last years, has received new impulse from the investigation of quantum systems which are chaotic in the classical limit. The important issue here is twofold. First, one would like to know if such quantum systems display some sort of chaotic behaviour; second, if this behaviour will provide foundations for quantum statistical mechanics. In other words, the problem is whether a $N$ - body isolated system, which, on the classical side, displays sufficiently strong chaotic properties, will, on the quantum side, give rise to conventional statistical properties, such as, e.g., the Bose-Einstein (BE) or Fermi-Dirac (FD) distributions [1]. The first problem has been widely investigated for single-particle systems; not so for many-body systems, essentially because such systems are not easily accessible to numerical investigations. Instead, little is known about the second problem.

Whereas early studies on the foundations of quantum statistical mechanics did not attach special importance to the structure of eigenstates, quantum ergodicity is nowadays associated with eigenstates being homogeneously spread, in an appropriate statistical sense, over the whole energetically allowed range. Much of our current understanding of the structure of eigenstates for classically chaotic systems is based on the study of single-particle systems, and on the analysis of eigenfunctions of Random Matrices, which have been often used as models for quantum chaos. In particular, suitably constructed Wigner Banded Random Matrices (WBRM) [2] are conjectured to preserve many of the spectral properties of "chaotic" Hamiltonian matrices [3]. For WBRM, several results are known [4], concerning spectrum statistics, structure of eigenstates and of the Local Spectral Density of States (also called Strength Function), and conditions for quantum ergodicity have been given [7]. These results provide paradigms of quantum ergodic behaviour, which should be tested on realistic Hamiltonians.

If quantum ergodicity can be established along such lines for quantum many-body systems, then the problem arises, whether it gives rise to some sort of statistical equilibrium. One would like to know whether the quantum averages of the occupation numbers of single-particle energy levels, taken over many-body ergodic eigenstates (or, more properly, over mixtures of eigenstates with energies lying in a narrow selected range), yield some statistically stable distribution law; if this is the case, how large must the number of particles be, in order that this distribution reproduces the conventional Fermi-Dirac or Bose-Einstein statistics?

This theoretical approach has been advocated in Refs. [8 10]; in this Letter we numerically investigate the just sketched theoretical issues on an isolated system of two interacting particles, which, under appropriate conditions, is classically chaotic in some energy region. This model has been proposed and investigated in 111 within the framework of quantum chaos; here we analyze it from the standpoint of statistical mechanics, and show that thermalization occurs in the classically chaotic energy region, in a sense that will be discussed below.

We first review some fundamental facts about its classical and quantum behavior. The model describes two coupled rotators, with angular momentum $\vec{L}, \vec{M}$ and Hamiltonian :

$$
H=H_{0}+V=\left(L_{z}+M_{z}\right)+L_{x} M_{x}
$$

It may be used to describe the interaction of quasispins in nuclear physics. Constants of motion are $H=E$, $L^{2}$ and $M^{2}$. It is worth to mention that in this form the dynamical variables $\vec{L}, \vec{M}$ are not canonical. 
The analysys of the surfaces of section reveals a large number of regular trajectories covering invariant tori when $L^{2}, M^{2}$ are both very small or very large $\mid 11$. To simplify the problem we set $L=M$. In such a case the most interesting situation occurs when $1<L<10$ where, depending on the energy value $E$, regular and chaotic regions coexist. Typically when $|E|$ is close to the maximum allowed energy $E_{\max }=L^{2}+1[1]$ trajectories are regular while for $E \simeq 0$ the islands of stability become very small and chaotic motion dominates.

Quantization follows standard rules, and angular momenta are quantized according to the relations $L^{2}=$ $M^{2}=\hbar^{2} l(l+1)$ where $l$ is an integer number. Therefore, for given $l$ the Hamiltonian is a finite matrix, and the semiclassical limit is recovered in the limit $l \rightarrow \infty$ and $\hbar \rightarrow 0$ keeping $L^{2}$ constant.

The matrix elements in the basis $\left|l_{z}, m_{z}\right\rangle$ have the form,

$$
\begin{gathered}
\left\langle l_{z}^{\prime}, m_{z}^{\prime}\left|H_{0}\right| l_{z}, m_{z}\right\rangle=\delta_{m_{z}, m_{z}^{\prime}} \delta_{l_{z}, l_{z}^{\prime}} \hbar\left(l_{z}+m_{z}\right) \\
\left\langle l_{z}^{\prime}, m_{z}^{\prime}|V| l_{z}, m_{z}\right\rangle=\frac{\hbar^{2}}{4} \delta_{m_{z}, m_{z}^{\prime} \pm 1} \delta_{l_{z}, l_{z}^{\prime} \pm 1} \times \\
\times\left[\left(l+l_{z}\right)\left(l-l_{z}+1\right)\left(l+m_{z}\right)\left(l-m_{z}+1\right)\right]^{1 / 2}
\end{gathered}
$$

with $l_{z}, m_{z}$ integers, $-l \leq l_{z}, m_{z} \leq l$.

The $z$-component of the total angular momentum $J_{z}=L_{z}+M_{z}$ (which is the unperturbed Hamiltonian $H_{0}$ ) obeys the selection rules $\Delta J_{z}=0, \pm 2 \hbar$, so the subspace spanned by the states with odd $J_{z}$ can be separated from that with $J_{z}$ even (there are no matrix elements for the transition between them). In what follows, we fix $J_{z}=H_{0}$ even (multiple of $\hbar$ ).

A key point in our approach is to represent the Hamiltonian in the symmetrized two-particle basis of noninteracting particles. This corresponds to the well-known "shell model" representation used in atomic and nuclear physics. Here, we restrict our considerations only to symmetric states with respect to the exchange of the two particles. In the symmetrized basis each set of states with fixed even $H_{0}$ has a degeneracy $l+1-\left|H_{0}\right| / 2 \hbar$, and the dimension of the Hamiltonian matrix is $N=(l+1)^{2}$. Finally, we reorder the matrix according to increasing unperturbed energy, and thus obtain a band matrix, with high sparsity within the band (each line has at most 5 elements).

Direct diagonalization of such a matrix gives the eigenfunctions $\psi_{n}\left(E_{m}\right)$ of the total Hamiltonian $H$ represented in the ordered symmetrized two-particle basis $|\mathbf{n}\rangle$. Here $\psi_{n}\left(E_{m}\right)$ is the $n$-th component of the eigenfunction having $E_{m}$ as eigenvalue. A detailed analysis of the structure of eigenstates, (which will be reported in detail elsewhere [12]) reveals that eigenstates which belong to the classical chaotic region are ergodic, in the sense that they fill a range of unperturbed energies, in a way which corresponds to the classical microcanonical distribution. Here we concentrate on the distribution of the occupation numbers of single-particle states.

The distribution $n_{s}$ of occupation numbers of single particle levels $s$ can be directly obtained from eigenfunctions. Given an eigenfunction $\psi_{n}\left(E_{m}\right)$ one can write:

$$
n_{s}\left(E_{m}\right)=\sum_{n}\left|\psi_{n}\left(E_{m}\right)\right|^{2}\left\langle\mathbf{n}\left|\hat{n}_{s}\right| \mathbf{n}\right\rangle
$$

where $\hat{n}_{s}$ is the occupation number operator. The term $\left\langle\mathbf{n}\left|\hat{n}_{s}\right| \mathbf{n}\right\rangle$ equals $0,1,2$ depending on how many particles are located on the specific single-particle level $s$. In Fig.1 some examples for the occupation numbers distribution (histograms on the left column) are given, together with the corresponding eigenstates (right column).
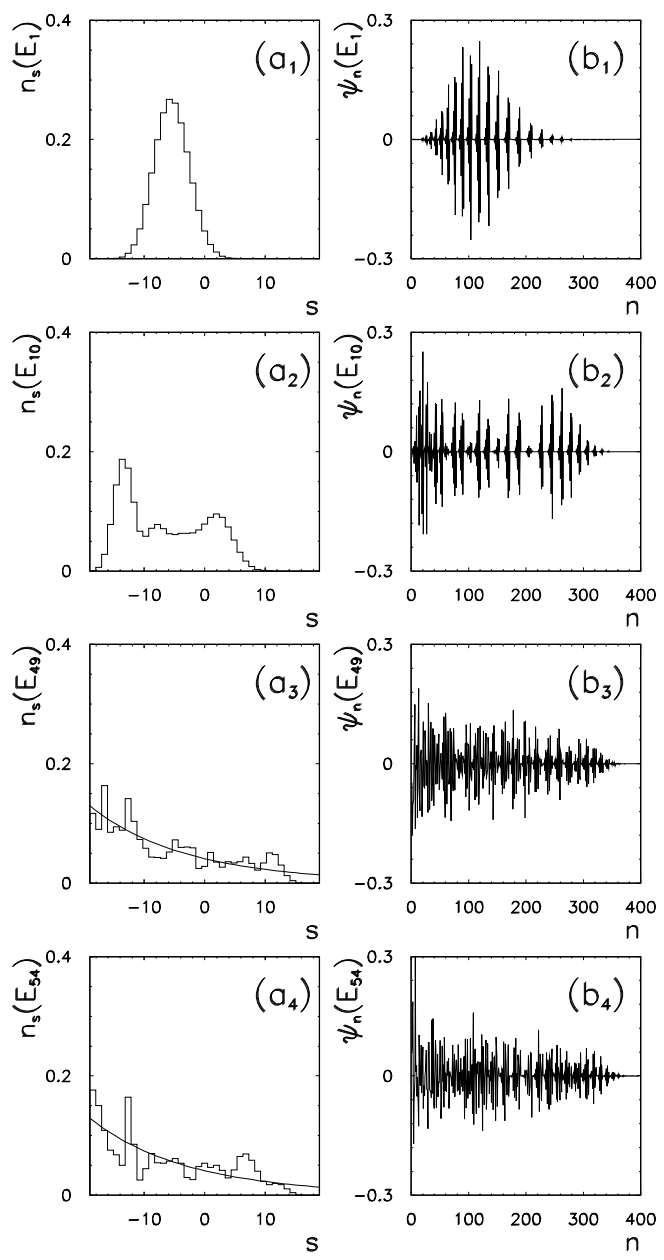

FIG. 1. Eigenfunctions (right column) and the corresponding occupation number distributions (left column) for the case $L=3.5, l=19 . \quad\left(a_{1}\right)$ and $\left(b_{1}\right)$ are for the ground state, $\left(a_{2}\right)$ and $\left(b_{2}\right)$ are for the $10^{t h}$ state (classically quasi-integrable), $\left(a_{3}\right)$ and $\left(b_{3}\right)$ are for the $49^{t h}$ state (with a chaotic phase space and $e_{m}=E_{m} / E_{\max }=-0.464$ ) and the full line is the best fit to the BE distribution with $\beta_{\text {fit }}=0.305$; $\left(a_{4}\right)$ and $\left(b_{4}\right)$ are for the $54^{t h}$ state (classically chaotic) with $e_{m}=-0.449, \beta_{\text {fit }}=0.310$ 
One can see a strong difference between the distributions obtained from "regular" eigenstates, belonging to the stable region $\left(b_{1}, b_{2}\right)$, and those obtained from ergodic ones, belonging to the chaotic region $\left(b_{3}, b_{4}\right)$. In the stable region close eigenstates in energy $E_{m}$ yield completely different distributions $n_{s}$ (see Fig.1 $\left(a_{1}, a_{2}\right)$ ), which means that there is no equilibrium in the statistical sense 10].

In contrast, in the classically chaotic region the form of the distribution $n_{s}$ is statistically stable with respect to the choice of a specific chaotic eigenstate (compare Fig.11 $\left.a_{3}, a_{4}\right)$.

In the standard thermodynamical treatment, temperature can be defined in a number of different ways, which are known to be equivalent in the thermodynamical limit. It is interesting to compute temperature for our system, following these different definitions, although our system is far from this limit. First of all we use the canonical expression:

$$
\langle E\rangle_{\beta_{c}}=\frac{\sum_{m} E_{m} e^{-\beta_{c} E_{m}}}{\sum_{m} e^{-\beta_{c} E_{m}}}
$$

where $E_{m}$ are the exact eigenenergies of the interacting system. The above relation between energy and temperature allows for standard thermodynamical description of our system. The solution $\beta_{c}(E)$ of equation (4) is shown in Fig.2 as a full curve.

For a system of $N$ non-interacting particles with total energy $E$, the canonical distribution is well-known to result, when $N$ is large, in the BE distribution for the occupation numbers: $n_{s}^{B E}=\left[e^{\beta(\hbar(s+l)-\mu)}-1\right]^{-1} \quad(s=$ $-l, \ldots, l)$ where $\mu$ is the chemical potential and $\beta$ is the inverse temperature. This expression is derived for an ideal gas (many non-interacting particles) in contact with a thermostat; in contrast, our system is isolated, with two interacting particles only. Nevertheless, recent analytical and numerical studies for random two-body interaction [8 10, suggest that conventional quantum statistics can appear even in isolated systems with relatively few particles, provided a proper renormalization of energy is taken.

As a simple comparison, we may consider the BE distribution as one-parameter fitting expression, taking into account the constraint set by the finite number of particles $\left(\sum_{s} n_{s}^{B E}=2\right)$. This allows to find the corresponding inverse temperature $\beta_{f i t}$, and from that the BE distribution presented in the left column of Fig.1 by full curves.

A different way of comparing numerical data with the $\mathrm{BE}$ distribution is to solve the following equations in the unknowns $\beta, \mu$ :

$$
\sum_{s=-l}^{l} n_{s}^{B E}=2, \sum_{s=-l}^{l} \hbar s n_{s}^{B E}=\mathcal{E}
$$

with $\mathcal{E}=\sum_{s} \hbar s n_{s}$, computed from numerical values of $n_{s}$ (note that $\mathcal{E}$ is different from the exact eigenenergy be- cause our model is strongly non-perturbative [12]). Doing so, and averaging over a number of chaotic eigenstates with close values inside small energy windows, we have found inverse temperatures $\beta_{B E}$ quite close to the previously obtained $\beta_{f i t}$ (compare crosses with full circles in Fig.2). The agreement between the numerical values of $\beta_{f i t}$ and $\beta_{B E}$ supports, on one hand, the significance of the fitting procedure with a $\mathrm{BE}$ distribution and, on the other hand, the validity of the BE distribution for isolated systems with few interacting particles via a proper renormalization of the energy $\mathcal{E}$, see [9,10].

We have then compared $\beta_{f i t}(E)$ with $\beta_{c}(E)$, where $E$ is the exact eigenenergy, and have found them to be significantly different. This is of course hardly surprising: even in the presence of ergodicity, with so few particles, one cannot expect coincidence of microcanonical and canonical averages, which still strongly resent of the particular choice of the interaction. In classical terms, the distribution of single-particle energy still depends on the particular shape of the energy surface, which is in turn determined by the particular interaction chosen.

A remarkably different result was obtained on "randomizing" our model, by replacing non zero off-diagonal matrix elements in the Hamiltonian by random variables with the same mean and variance as in the exact dynamical model (11). Whereas $\beta_{c}$ is left practically unchanged by this replacement, $\beta_{f i t} \simeq \beta_{B E}$ considerably changes, and it comes quite close to $\beta_{c}$. In our understanding, the reason of this striking result is that the random model (which has no smooth classical limit) uses a much more "generic" form of the perturbation than the dynamical one; in addition, the dependence on the specific interaction is further weakened by the (matrix) ensemble averaging. As a result, our data indicate that the conventional canonical distribution of occupation numbers may appear even in an isolated system with a quite small number of particles with random interactions, where the dynamical correlations, which prevent a similar results in the classical model, are negligible.

Other different definitions of temperature have been widely discussed in application to complex nuclei [13] in the context of the onset of thermalization. It was in particular shown that these definitions of temperature give the same result in realistic shell models of nuclei. This fact was connected with the onset of "true" thermalization. However, as was shown in [10], when the number of interacting particles is small, one can get different values of temperature depending on the particular definition, even in the equilibrium region, where a statistical description is legitimate. In this connection we have also compared the temperatures found from the BE distribution with the standard thermodynamical inverse temperature defined by, $\beta_{T}=d \ln \rho / d E$ where $\rho$ is the density of states of the total Hamiltonian. Both for the dynamical and the random model, a Gaussian fit provides an 
excellent approximation to actual data for the density of states [14]. We have used this very fit in computing $\beta_{T}$, obtaining practically the same result in both cases. The function $\beta_{T}(E)$ is different from $\beta_{c}(E)$, but it comes closer and closer to it near the center of the spectrum (where both temperatures are infinite). For the dynamical model, $\beta_{T}(E)$ turns out to be completely different from both $\beta_{c}$ and $\beta_{f i t} \simeq \beta_{B E}$ (see dotted line in Fig.2). This means that for small number of particles the above definition of $\beta_{T}$ is irrelevant to the distribution of occupation numbers [10]. For the random model, $\beta_{T}$ is not far from $\beta_{f i t}(E)$ at high temperatures (where $\beta_{T}$ and $\beta_{c}$ tend to coincide), but deviates from it at smaller temperatures. We do not push our comparison of $\beta_{f i t}(E)$ and $\beta_{c}$ to smaller temperatures than shown in Fig.2, because near the edges of the spectrum, where the density of states is small, the eigenstates are not fully chaotic any more (note that in the dynamical model the motion becomes increasingly regular there).

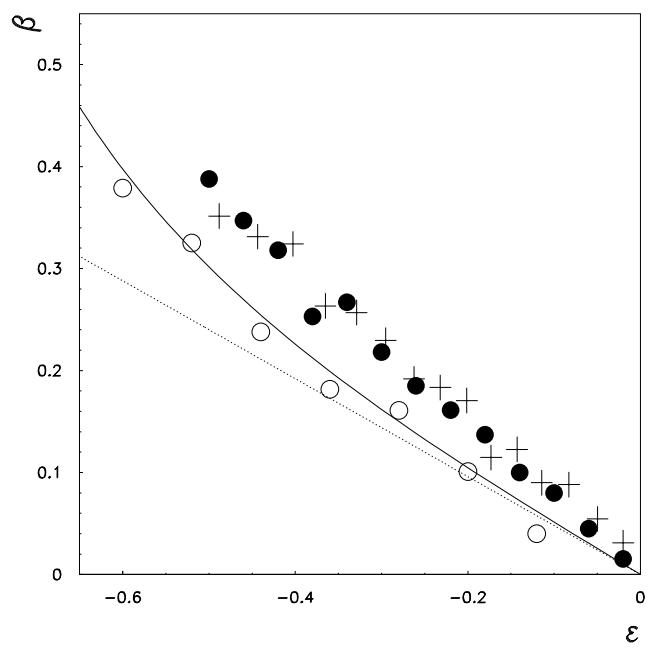

FIG. 2. Dependence of the different definitions of inverse temperature $\beta$ on the rescaled energy $\varepsilon=E / E_{\text {max }}$; full curve: $\beta_{c}$, dotted curve : $\beta_{T}$, full circles : $\beta_{f i t}$, crosses : $\beta_{B E}$. Open circles are $\beta_{f i t}$ for the random model.

In conclusion, we have studied a dynamical model of two-interacting Boson particles in a finite dimensional Hilbert space. We have shown that, in the shell model representation, the structure of exact eigenstates can be directly related to the onset of equilibrium for the occupation numbers $n_{s}$ of single-particle states. Specifically, for the eigenstates corresponding to classical chaotic motion, an equilibrium distribution for $n_{s}$ occurs which allows for a statistical description of the model. In contrast, "regular" eigenstates results in extremely non- generic fluctuations of $n_{s}$ for small changes of the energy, thus invalidating any statistical approach. For chaotic eigenstates, the distribution of occupation numbers can be approximately described by the Bose-Einstein distribution, although the system is isolated and consists of two particles only. In this case a strong enough interaction plays the role of a heat bath, thus leading to thermalization. In spite of this, the minimal number of particles prevents the canonical distribution from describing our dynamical system even if a surprising agreement with the canonical distribution is recovered in the corresponding random model.

The authors are grateful to V.Flambaum for fruitful discussion. (FMI) thanks with pleasure the colleagues of the University of Milan at Como for the hospitality during his visit when this work was done; he acknowledges the support from the Grant of the Cariplo Foundation for Research and partial support from the INTAS Grant No. 94-2058.

[1] G.Casati " On the Foundations of Equilibrium Quantum Statistical Mechanics" preprint 1997.

[2] E. Wigner, Ann. Math. 62, 548 (1955); 65, 203 (1957).

[3] M. Feingold, D.M. Leitner and O. Piro, Phys. Rev. A 39, 6507 (1989).

[4] M. Feingold, D. Leitner and M. Wilkinson, Phys. Rev. Lett. 66, 986 (1991); J. Phys. A 24, 1751 (1991); D.M. Leitner and M. Feingold, J. Phys. A 26, 7367 (1993).

[5] G. Casati, B.V. Chirikov, I. Guarneri and F.M. Izrailev, Phys. Rev. E 48, R1613 (1993).

[6] Y.V. Fyodorov, O.A. Chubykalo, F.M. Izrailev and G. Casati, Phys. Rev. Lett. 76, 1603 (1996).

[7] G. Casati, B.V. Chirikov, I. Guarneri and F.M. Izrailev, Phys. Lett. A 223, 430 (1996).

[8] V.V. Flambaum, F.M. Izrailev and G. Casati, Phys. Rev. E 53, 2136 (1996).

[9] V.V. Flambaum and F.M. Izrailev, Phys. Rev. E 55, R13, (1997).

[10] V.V. Flambaum and F.M. Izrailev, cond-mat/9707016, to appear in Phys. Rev. E (1997) .

[11] M. Feingold and A. Peres, Physica 9D, 433 (1983), M. Feingold, N. Moiseyev and A. Peres, Phys. Rev. A 30, 509 (1984); A. Peres, Phys. Rev. Lett. 53, 1711 (1984).

[12] F. Borgonovi, I. Guarneri and F.M. Izrailev in preparation

[13] M.Horoi, V.Zelevinsky and B.A.Brown, Phys. Rev. Lett., 74, 26 (1995); V. Zelevinsky, B.A. Brown, M. Horoi and N. Frazier, Phys. Rep. 276, 85 (1996).

[14] J.B. French and S.S.M. Wong, Phys. Lett. B 35, 5 (1970); O. Bohigas and J. Flores, Phys. Lett. B 34, 261 (1971). 